\title{
The Minor Crossing Number of Graphs with an Excluded Minor
}

\author{
Drago Bokal \\ Department of Combinatorics and Optimization \\ University of Waterloo \\ Waterloo, Canada \\ dbokal@uwaterloo.ca \\ Gašper Fijavž \\ Faculty of Computer and Information Science \\ University of Ljubljana \\ Ljubljana, Slovenia \\ gasper.fijavz@fri.uni-lj.si \\ David R. Wood* \\ Departament de Matemática Aplicada II \\ Universitat Politècnica de Catalunya \\ Barcelona, Spain \\ david.wood@upc.edu
}

Submitted: Dec 7, 2006; Accepted: Dec 7, 2007; Published: Jan 1, 2008

Mathematics Subject Classifications:

05C62 (graph representations), 05C10 (topological graph theory), 05C83 (graph minors)

\begin{abstract}
The minor crossing number of a graph $G$ is the minimum crossing number of a graph that contains $G$ as a minor. It is proved that for every graph $H$ there is a constant $c$, such that every graph $G$ with no $H$-minor has minor crossing number at most $c|V(G)|$.
\end{abstract}

* The research of David Wood is supported by a Marie Curie Fellowship of the European Community under contract 023865, and by the projects MCYT-FEDER BFM2003-00368 and Gen. Cat 2001SGR00224. 


\section{Introduction}

The crossing number of a graph ${ }^{1} G$, denoted by $\operatorname{cr}(G)$, is the minimum number of crossings in a drawing ${ }^{2}$ of $G$ in the plane; see $[13,28,29,37,48-50]$ for surveys. The crossing number is an important measure of the non-planarity of a graph [48], with applications in discrete and computational geometry [27, 47] and VLSI circuit design [3, 20, 21]. In information visualisation, one of the most important measures of the quality of a graph drawing is the number of crossings [34-36].

We now outline various aspects of the crossing number that have been studied. First note that computing the crossing number is $\mathcal{N} \mathcal{P}$-hard [15], and remains so for simple cubic graphs [19, 31]. Moreover, the exact or even asymptotic crossing number is not known for specific graph families, such as complete graphs [40], complete bipartite graphs $[23,38,40]$, and Cartesian products $[1,5,6,17,39]$. Given that the crossing number seems so difficult, it is natural to focus on asymptotic bounds rather than exact values. The 'crossing lemma', conjectured by Erdős and Guy [13] and first proved by Leighton [20] and Ajtai et al. [2], gives such a lower bound. It states that for some constant $c, \operatorname{cr}(G) \geq$ $c\|G\|^{3} /|G|^{2}$ for every graph $G$ with $\|G\| \geq 4|G|$. See $[22,25]$ for recent improvements. Other general lower bound techniques that arose out of the work of Leighton [20, 21] include the bisection/cutwidth method [11, 26, 45, 46] and the embedding method [44, 45]. Upper bounds on the crossing number of general families of graphs have been less studied. One example, by Pach and Tóth [30], says that graphs $G$ of bounded genus and bounded degree have $\mathcal{O}(|G|)$ crossing number. See $[9,12]$ for extensions. The present paper also focuses on crossing number upper bounds.

Graph minors ${ }^{3}$ are a widely used structural tool in graph theory. So it is inviting to explore the relationship between minors and the crossing number. One impediment is that the crossing number is not minor-monotone; that is, there are graphs $G$ and $H$ with $H$ a minor of $G$, for which $\operatorname{cr}(H)>\operatorname{cr}(G)$. Nevertheless, following an initial paper by Robertson and Seymour [41], there have been a number of recent papers on the relationship between crossing number and graph minors $[7,8,14,16,18,19,24,31,51]$. For example, Wood and Telle [51] proved the following upper bound (generalising the

\footnotetext{
${ }^{1}$ We consider finite, undirected, simple graphs $G$ with vertex set $V(G)$ and edge set $E(G)$. Let $|G|:=|V(G)|$ and $\|G\|:=|E(G)|$. Let $\Delta(G)$ be the maximum vertex degree of $G$.

${ }^{2} \mathrm{~A}$ drawing of a graph represents each vertex by a distinct point in the plane, and represents each edge by a simple closed curve between its endpoints, such that the only vertices an edge intersects are its own endpoints, and no three edges intersect at a common point (except at a common endpoint). A crossing is a point of intersection between two edges (other than a common endpoint). A graph is planar if it has a crossing-free drawing.

${ }^{3}$ Let $v w$ be an edge of a graph $G$. Let $G^{\prime}$ be the graph obtained by identifying the vertices $v$ and $w$, deleting loops, and replacing parallel edges by a single edge. Then $G^{\prime}$ is obtained from $G$ by contracting $v w$. A graph $H$ is a minor of a graph $G$ if $H$ can be obtained from a subgraph of $G$ by contracting edges. A family of graphs $\mathcal{F}$ is minor-closed if $G \in \mathcal{F}$ implies that every minor of $G$ is in $\mathcal{F}$. $\mathcal{F}$ is proper if it is not the family of all graphs. A deep theorem of Robertson and Seymour [43] states that every proper minor-closed family can be characterised by a finite family of excluded minors. Every proper minor-closed family is a subset of the $H$-minor-free graphs for some graph $H$. We thus focus on minor-closed families with one excluded minor.
} 
above-mentioned results in $[9,12,30]$ for graphs of bounded genus).

Theorem 1 ([51]). For every graph $H$ there is a constant $c=c(H)$, such that every $H$-minor-free graph $G$ has crossing number $\operatorname{cr}(G) \leq c \Delta(G)^{2}|G|$.

\subsection{Minor Crossing Number}

Bokal et al. [8] defined the minor crossing number of a graph $G$, denoted by $\operatorname{mcr}(G)$, to be the minimum crossing number of a graph that contains $G$ as a minor. The main motivation for this definition is that for every constant $c$, the family of graphs $G$ for which $\operatorname{mcr}(G) \leq c$ is closed under taking minors. Moreover, the minor crossing number corresponds to a natural style of graph drawing, in which each vertex is drawn as a tree. Bokal et al. [7] proved a number of lower bounds on the minor crossing number that parallel the lower bound techniques of Leighton. The main result of this paper is to prove the following upper bound, which is an analogue of Theorem 1 for the minor crossing number (without the dependence on the maximum degree).

Theorem 2. For every graph $H$ there is a constant $c=c(H)$, such that every $H$-minorfree graph $G$ has minor crossing number $\operatorname{mcr}(G) \leq c|G|$.

The restriction to graphs with an excluded minor in Theorem 2 is unavoidable in the sense that $\operatorname{mcr}\left(K_{n}\right) \in \Theta\left(n^{2}\right)$. The linear dependence in Theorem 2 is best possible since $\operatorname{mcr}\left(K_{3, n}\right) \in \Theta(n)$. Both these bounds were established by Bokal et al. [8]. An elegant feature of Theorem 2 and the minor crossing number is that there is no dependence on the maximum degree, unlike in Theorem 1, where some dependence on the maximum degree is unavoidable. In particular, the complete bipartite graph $K_{3, n}$ has no $K_{5}$-minor and has $\Theta\left(n^{2}\right)$ crossing number $[23,38]$.

\section{Planar Decompositions}

It is widely acknowledged that the theory of crossing numbers needs new ideas. Some tools that have been recently developed include 'meshes' [39], 'arrangements' [1], 'tile drawings' [4, 32, 32, 33], and the 'zip product' [4-6]. A feature of the proof of Theorem 1 by Wood and Telle [51] is the use of 'planar decompositions' as a new tool for studying the crossing number. Planar decompositions are also the key component in the proof of Theorem 2 in this paper.

Let $G$ and $D$ be graphs, such that each vertex of $D$ is a set of vertices of $G$ (called a $b a g)$. Note that we allow distinct vertices of $D$ to be the same set of vertices in $G$; that is, $V(D)$ is a multiset. For each vertex $v$ of $G$, let $D(v)$ be the subgraph of $D$ induced by the bags that contain $v$. Then $D$ is a decomposition of $G$ if:

- $D(v)$ is connected and nonempty for each vertex $v$ of $G$, and 
- $D(v)$ and $D(w)$ touch $^{4}$ for each edge $v w$ of $G$.

Decompositions, when $D$ is a tree, were first studied in detail by Robertson and Seymour [42]. Diestel and Kühn $[10]^{5}$ first generalised the definition for arbitrary graphs $D$.

We measure the 'complexity' of a graph decomposition $D$ by the following parameters. The width of $D$ is the maximum cardinality of a bag. The order of $D$ is the number of bags. The degree of $D$ is the maximum degree of the graph $D$. The decomposition $D$ is planar if the graph $D$ is planar.

Diestel and Kühn [10] observed that decompositions generalise minors in the following sense.

Lemma 1 ([10]). A graph $G$ is a minor of a graph $D$ if and only if a graph isomorphic to $D$ is a decomposition of $G$ with width 1 .

Wood and Telle [51] describe a number of tools for manipulating decompositions, such as the following lemma for composing two decompositions.

Lemma 2 ([51]). Suppose that $D$ is a decomposition of a graph $G$ with width $k$, and that $J$ is a decomposition of $D$ with width $\ell$. Then $G$ has a decomposition isomorphic to $J$ with width $k \ell$.

Lemma 2 has the following special case, which follows from Lemma 1.

Lemma 3. If a graph $G_{1}$ is a minor of a graph $G_{2}$, and $J$ is a decomposition of $G_{2}$ with width $\ell$, then some graph isomorphic to $J$ is a decomposition of $G_{1}$ with width $\ell$.

The next tool by Wood and Telle [51] reduces the order of a planar decomposition at the expense of increasing the width.

Lemma 4 ([51]). Suppose that a graph $G$ has a planar decomposition $D$ of width $k$ and order at most $c|G|$ for some $c \geq 1$. Then $G$ has a planar decomposition of width $c^{\prime} k$ and order $|G|$, for some $c^{\prime}$ depending only on $c$.

Converse to Lemma 4, we now show that the width and degree of a planar decomposition can be reduced at the expense of increasing the order.

Lemma 5. If a graph $G$ has a planar decomposition $D$ of width $k$, then $G$ has:

(a) a planar decomposition $D_{1}$ of width $k$, order $\left|D_{1}\right|<6|D|$ and degree $\Delta\left(D_{1}\right) \leq 3$,

(b) a planar decomposition $D_{2}$ of width 2 , order $\left|D_{2}\right|<3 k(k+1)|D|$ and degree $\Delta\left(D_{2}\right) \leq 4$,

(c) a planar decomposition $D_{3}$ of width 2 , order $\left|D_{3}\right|<6 k^{2}|D|$ and degree $\Delta\left(D_{3}\right) \leq 3$.

\footnotetext{
${ }^{4}$ Let $A$ and $B$ be subgraphs of a graph $G$. Then $A$ and $B$ intersect if $V(A) \cap V(B) \neq \emptyset$, and $A$ and $B$ touch if they intersect or $v \in V(A)$ and $w \in V(B)$ for some edge $v w$ of $G$.

${ }^{5} \mathrm{~A}$ decomposition was called a connected decomposition by Diestel and Kühn [10].
} 
Proof. For the clarity of presentation, we assume that all the bags of $D$ have width $k$, although this assumption is not used in the proof. We also assume that $D$ has minimum degree at least 3 ; the reader can easily adapt the construction to vertices of degrees 1 and 2. (Alternatively, we can augment $D$ to have minimum degree 3 by adding new edges, whenever $D$ has at least 4 vertices.) Fix an embedding of $D$ in the plane.

First we prove (a). Let $D_{1}$ be the graph with two vertices $X_{e}$ and $Y_{e}$ for every edge $e=X Y \in E(D)$, where each bag $X_{e}$ is a copy of $X$. We say that $X_{e}$ belongs to $X$. Add the edge $X_{e} Y_{e}$ to $D_{1}$ for each edge $e=X Y \in E(D)$. Add the edge $X_{e} X_{f}$ to $D_{1}$ whenever the edges $e$ and $f$ are consecutive in the cyclic order of edges incident to a bag $X$ in $D$.

As illustrated in Figure 1(b), each bag $X$ is thus replaced by a cycle in $D_{1}$, each vertex of which has one more incident edge in $D_{1}$. Thus $D_{1}$ is a planar graph with maximum degree 3 and order $\left|D_{1}\right|=2\|D\|$ (after adding edges to $D$, if necessary). Since $D$ is planar, $\|D\| \leq 3|D|-6$ and so $\left|D_{1}\right| \leq 6|D|-12$. Since the set of bags of $D_{1}$ that belong to a specific bag of $D$ induces a connected (cycle) subgraph of $D_{1}$, and $D(v)$ is a connected subgraph of $D$ for each vertex $v$ of $G, D_{1}(v)$ is a connected subgraph of $D_{1}$.

We now prove that $D_{1}(v)$ and $D_{1}(w)$ touch for each edge $v w$ of $G$. If $v$ and $w$ are in a common bag $X$ of $D$, then $v$ and $w$ are in every bag $X_{e}$ of $D_{1}$. Otherwise, $v \in X$ and $w \in Y$ for some edge $e=X Y$ of $D$, in which case $v \in X_{e}, w \in Y_{e}$, and $X_{e} Y_{e}$ is an edge of $D_{1}$. Thus $D_{1}(v)$ and $D_{1}(w)$ touch. Therefore $D_{1}$ is a planar decomposition of $G$. This completes the proof of (a).

Now we prove (b). Fix an arbitrary linear order $\preceq$ on $V(G)$, and arbitrarily orient the edges of $D$. For each arc $e=\overrightarrow{X Y}$ of $D$, orient the edge $X_{e} Y_{e}$ of $D_{1}$ from $X_{e}$ to $Y_{e}$.

Informally speaking, we now construct a planar decomposition $D_{2}$ from $D_{1}$ by replacing each bag $X_{e}$ of $D_{1}$ by a set of $\left(\begin{array}{c}k+1 \\ 2\end{array}\right)$ bags, each of width 1 or 2 , that form a wedge pattern, as illustrated in Figure 1(c). Depending on whether $e$ is incoming or outgoing at $X$, the wedge is reflected appropriately to ensure the planarity of $D_{2}$.

Now we define $D_{2}$ formally. Consider a bag $X=\left\{v_{1}, v_{2}, \ldots, v_{k}\right\}$ of $D$, where $v_{1} \prec$ $v_{2} \prec \cdots \prec v_{k}$. For each pair of vertices $v_{i}, v_{j}$ in $X$, and for each edge $e$ incident to $X$, add a bag labelled $\left\{v_{i}, v_{j}\right\}_{X_{e}}$ to $D_{2}$, where $\left\{v_{i}, v_{j}\right\}_{X_{e}}$ is a copy of $\left\{v_{i}, v_{j}\right\}$. (The bag $\left\{v_{i}, v_{i}\right\}_{X_{e}}$ is a singleton $\left\{v_{i}\right\}$.) We say that $\left\{v_{i}, v_{j}\right\}_{X_{e}}$ belongs to $X_{e}$ and to $X$. Thus there are $\left(\begin{array}{c}k+1 \\ 2\end{array}\right)$ bags that belong to each bag of $D_{1}$. Hence $\left|D_{2}\right| \leq\left(\begin{array}{c}k+1 \\ 2\end{array}\right)\left|D_{1}\right|<3 k(k+1)|D|$. Add an edge in $D_{2}$ between the bags $\left\{v_{i}, v_{j}\right\}_{X_{e}}$ and $\left\{v_{i}, v_{j+1}\right\}_{X_{e}}$ for $1 \leq i \leq k$ and $1 \leq j \leq k-1$. As illustrated in Figure 1(c), the subgraph of $D_{2}$ induced by the bags that belong to each bag $X_{e}$ of $D_{1}$ form a planar grid-like graph.

Consider two edges $e=X Y$ and $f=X Z$ of $D$ that are consecutive in the cyclic order of edges incident to a bag $X$ of $D$ (defined by the planar embedding). Without loss of generality, $X Z$ is clockwise from $X Y$. We now add edges to $D_{2}$ between certain bags that belong to $X_{e}$ and $X_{f}$ depending on the orientations of the edges $X Y$ and $X Z$. Since $D$ has minimum degree at least 3 , the bags corresponding to $X$ form a cycle in $D_{2}$. (For $D$-vertices of degree less than 3 , the construction is slightly different; we leave the details of $\operatorname{deg}_{D}(v) \leq 2$ to the reader.) For $1 \leq i \leq k$, let $P_{i}$ be the bag $\left\{v_{i}, v_{k}\right\}_{X_{e}}$ if $\overrightarrow{X Y}$ and $\left\{v_{i}, v_{i}\right\}_{X_{e}}$ if $\overrightarrow{Y X}$, and let $Q_{i}$ be the bag $\left\{v_{i}, v_{i}\right\}_{X_{f}}$ if $\overrightarrow{X Z}$ and $\left\{v_{i}, v_{k}\right\}_{X_{f}}$ if $\overrightarrow{Z X}$. Add an edge between $P_{i}$ and $Q_{i}$ for $1 \leq i \leq k$. As illustrated in Figure 1(c), the subgraph of $D_{2}$ 
(a) $D$

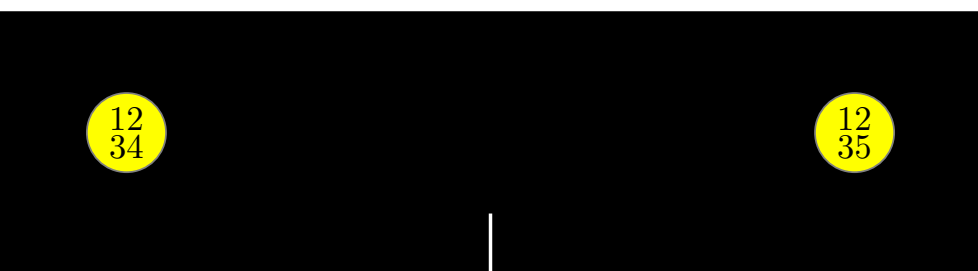

(b) $D_{1}$
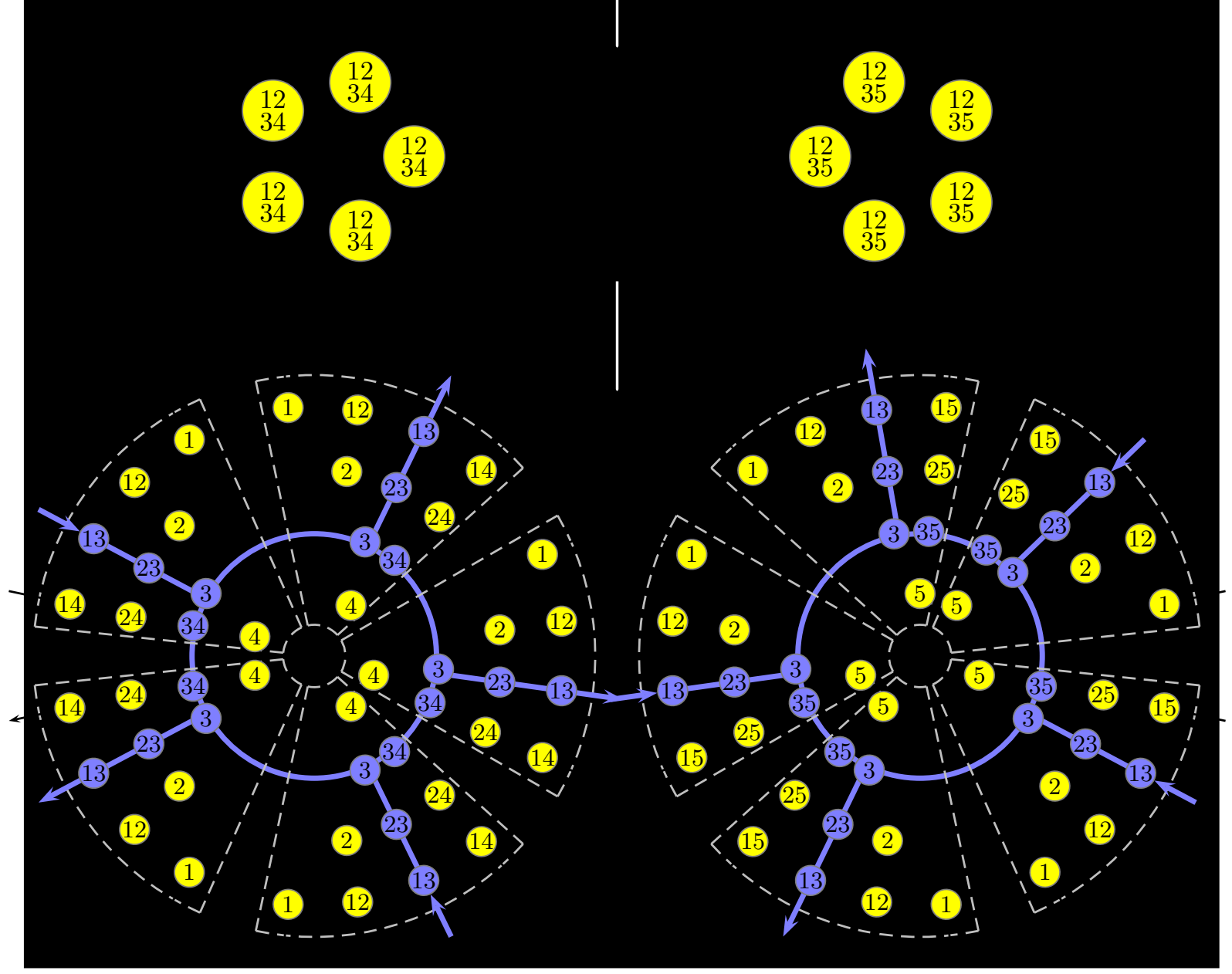

Figure 1: (a) The planar decomposition $D$. (b) The planar decomposition $D_{1}$ obtained from $D$ by replacing each bag of degree $d$ by $d$ bags of degree 3. (c) The planar decomposition $D_{2}$ obtained from $D_{1}$ by replacing each bag of width $k$ by $\left(\begin{array}{c}k+1 \\ 2\end{array}\right)$ bags of width 2 . The subgraph $D_{2}(3)$ is highlighted.

induced by the bags that belong to each bag $X$ of $D$ is planar.

Now consider an edge $e=\overrightarrow{X Y}$ of $D$, where $X=\left\{v_{1}, v_{2}, \ldots, v_{k}\right\}$ with $v_{1} \prec v_{2} \prec \cdots \prec$ $v_{k}$, and $Y=\left\{w_{1}, w_{2}, \ldots, w_{k}\right\}$ with $w_{1} \prec w_{2} \prec \cdots \prec w_{k}$. Whenever $v_{i}=w_{j}$, add an edge between $\left\{v_{1}, v_{i}\right\}_{X_{e}}$ and $\left\{w_{1}, w_{j}\right\}_{Y_{e}}$ to $D_{2}$. This completes the construction of $D_{2}$. Observe that the bags $\left\{v_{1}, v_{1}\right\}_{X_{e}},\left\{v_{1}, v_{2}\right\}_{X_{e}}, \ldots,\left\{v_{1}, v_{k}\right\}_{X_{e}}$ are ordered clockwise on the 
outer face of the subgraph of $D_{2}$ induced by the bags belonging to $X$. Similarly, the bags $\left\{w_{1}, w_{1}\right\}_{Y_{e}},\left\{w_{1}, w_{2}\right\}_{Y_{e}}, \ldots,\left\{w_{1}, w_{k}\right\}_{Y_{e}}$ are ordered anticlockwise on the outer face of the subgraph of $D_{2}$ induced by the bags belonging to $Y$. Thus these edges do not introduce any crossings in $D_{2}$, as illustrated in Figure 1(c).

We now prove that each subgraph $D_{2}(v)$ is a nonempty connected subgraph of $D_{2}$ for each vertex $v$ of $G$. Say $v$ is in a bag $X=\left\{v_{1}, v_{2}, \ldots, v_{k}\right\}$ of $D$, with $v_{1} \prec v_{2} \prec \cdots \prec v_{k}$ and $v=v_{i}$. Observe that the set of bags $\left\{\left\{v_{i}, v_{j}\right\}_{X_{e}}: v_{j} \in X \in e \in E(D), i \leq j\right\}$ forms a cycle in $D_{2}$ (drawn as a circle in Figure 1(c)), and for each edge $e$ incident to $X$, the bags $\left\{\left\{v_{i}, v_{j}\right\}_{X_{e}}: v_{j} \in X \in e \in E(D), j \leq i\right\}$ form a path between $\left\{v_{1}, v_{i}\right\}_{X_{e}}$ and $\left\{v_{i}, v_{i}\right\}_{X_{e}}$, where it attaches to this cycle. Thus the set of bags in $D_{2}$ that belong to $X$ and contain $v$ forms a connected subgraph of $D_{2}$. For each edge $e=X Y$ of $D$ with $v \in X \cap Y$, there is an edge in $D_{2}$ (between some bags $\left\{v_{1}, v_{i}\right\}_{X_{e}}$ and $\left\{w_{1}, w_{j}\right\}_{Y_{e}}$ ) that connects the set of bags that belong to $X$ and contain $v$ with the set of bags that belong to $Y$ and contain $v$. Thus $D_{2}(v)$ is connected since $D(v)$ is connected.

We now prove that $D_{2}(v)$ and $D_{2}(w)$ touch for each edge $v w$ of $G$. If $v$ and $w$ are in a common bag $X$ of $D$, then $v$ and $w$ are in every bag $\{v, w\}_{X_{e}}$ of $D_{1}$. Otherwise, $v \in X$ and $w \in Y$ for some edge $e=X Y$ of $D$, in which case $v$ and $w$ are in adjacent bags $\left\{v_{1}, v\right\}_{X_{e}}$ and $\left\{w_{1}, w\right\}_{Y_{e}}$, for appropriate vertices $v_{1}$ and $w_{1}$. Thus $D_{2}(v)$ and $D_{2}(w)$ touch. Therefore $D_{2}$ is a decomposition of $G$. Observe that $\Delta\left(D_{2}\right) \leq 4$. This completes the proof of (b).

Now we prove (c). Construct a planar decomposition $D_{3}$ from $D_{2}$ by the following operation applied to each bag $W$ of $D_{2}$ with degree 4. Say the neighbours of $W$ are $Z_{1}, Z_{2}, Z_{3}, Z_{4}$ in clockwise order in the embedding of $D_{2}$. Replace $W$ by two bags $W_{1}$ and $W_{2}$, both copies of $W$, where $W_{1}$ is adjacent to $W_{2}, Z_{1}, Z_{2}$, and $W_{2}$ is adjacent to $W_{1}, Z_{3}, Z_{4}$. Clearly $D_{3}$ is a planar decomposition of $G$ with maximum degree 3 . For each bag $X_{e}$ of $D_{1}$, there are $k$ bags of degree 3 in $D_{2}$ and $\frac{1}{2} k(k-1)$ bags of degree 4 that belong to $X_{e}$. Since each bag of degree 4 in $D_{2}$ is replaced by two bags in $D_{3}$, there are $k+2\left(\frac{1}{2} k(k-1)\right)=k^{2}$ bags in $D_{3}$ that belong to $X_{e}$. Thus $\left|D_{3}\right| \leq 2 k^{2}\|D\|<6 k^{2}|D|$. This completes the proof of (c).

Note that the upper bound of $\left|D_{1}\right| \leq 6|D|$ in Lemma 5 (a) can be improved to $\left|D_{1}\right| \leq$ $4|D|$ by replacing each bag of degree $d$ by $d-2$ bags of degree 3, as illustrated in Figure 2 . We omit the details.

\section{Planar Decompositions and Crossing Number}

In this section we review some of the results by Wood and Telle [51] that link planar decompositions and crossing number.

Lemma 6 ([51]). If $D$ is a planar decomposition of a graph $G$ with width $k$, then $G$ has crossing number

$$
\operatorname{cr}(G) \leq k(k+1) \Delta(G)^{2}|D| .
$$




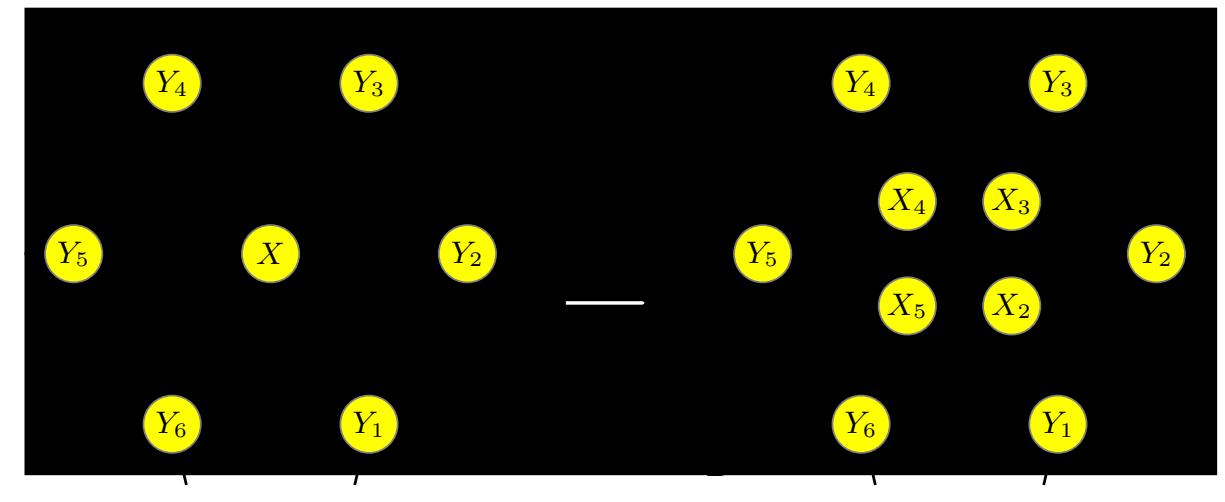

Figure 2: Replacing a bag of degree 6 by four bags of degree 3 .

Lemma 7 ([51]). For every graph $H$ there is an integer $k=k(H)$, such that every $H$-minor-free graph $G$ has a planar decomposition of width $k$ and order $|G|$.

Observe that Lemmas 6 and 7 imply Theorem 1. The next lemma is converse to Lemma 6.

Lemma $8([51])$. Every graph $G$ has a planar decomposition of width 2 and order $|G|+$ $\operatorname{cr}(G)$.

We have the following characterisation of graphs with linear crossing number.

Theorem 3 ([51]). The following are equivalent for a graph $G$ of bounded degree:

1. $\operatorname{cr}(G) \leq c_{1}|G|$ for some constant $c_{1}$,

2. $G$ has a planar decomposition with width $c_{2}$ and order $|G|$ for some constant $c_{2}$,

3. $G$ has a planar decomposition with width 2 and order $c_{3}|G|$ for some constant $c_{3}$.

Proof. Lemma 8 implies that $(1) \Rightarrow(3)$. Lemma 4 implies that $(3) \Rightarrow(2)$. Lemma 6 implies that $(2) \Rightarrow(1)$.

Note that Lemma $5(\mathrm{c})$ provides a more direct proof that $(2) \Rightarrow(3)$ in Theorem 3 (without the dependence on degree).

\section{Planar Decompositions and Minor Crossing Number}

Lemma 6 can be extended to give the following upper bound on the minor crossing number. Basically we replace the dependence on $\Delta(G)$ in Lemma 6 by $\Delta(D)$. 
Lemma 9. If $D$ is a planar decomposition of a graph $G$ with width $k$, then $G$ has minor crossing number

$$
\operatorname{mcr}(G)<k^{3}(k+1)(\Delta(D)+1)^{2}|D| .
$$

Proof. Let $G^{\prime}$ be the graph with one vertex for each occurrence of a vertex of $G$ in a bag of $D$. Consider a vertex $x$ of $G^{\prime}$ in bag $X$ and a distinct vertex $y$ of $G^{\prime}$ in bag $Y$. Connect $x$ and $y$ by an edge in $G^{\prime}$ if and only if $X=Y$ or $X Y$ is an edge of $D$. ( $G^{\prime}$ is a subgraph of the lexicographic product $D\left[K_{k}\right]$.) For each vertex $v$ of $G$, the copies of $v$ form a connected subgraph of $G^{\prime}$, since $D(v)$ is a connected subgraph of $D$. Since $D(v)$ and $D(w)$ touch for each edge $v w$ of $G$, some copy of $v$ is adjacent to some copy of $w$. Thus $G$ is a minor of $G^{\prime}$, and $\operatorname{mcr}(G) \leq \operatorname{cr}\left(G^{\prime}\right)$. Moreover, $D$ defines a planar decomposition of $G^{\prime}$ with width $k$. By Lemma 6 applied to $G^{\prime}$,

$$
\operatorname{mcr}(G) \leq \operatorname{cr}\left(G^{\prime}\right) \leq k(k+1) \Delta\left(G^{\prime}\right)^{2}|D|
$$

A neighbour of a vertex $x$ of $G^{\prime}$ is in the same bag as $x$ or is in a neighbouring bag. Thus $\Delta\left(G^{\prime}\right) \leq(\Delta(D)+1) k-1$. Thus

$$
\operatorname{mcr}(G)<k(k+1)((\Delta(D)+1) k)^{2}|D|=k^{3}(k+1)(\Delta(D)+1)^{2}|D| .
$$

Lemmas 9 and 5(a) imply that if $D$ is a planar decomposition of a graph $G$ with width $k$, then $G$ has minor crossing number in $\mathcal{O}\left(k^{4}|D|\right)$. This bound can be improved by further transforming the decomposition into a decomposition with width 2 . In particular, Lemmas 9 and 5(b) imply:

Lemma 10. If $D$ is a planar decomposition of a graph $G$ with width $k$, then $G$ has minor crossing number

$$
\operatorname{mcr}(G)<2^{3}(2+1)(4+1)^{2} 3 k(k+1)|D|=1800 k(k+1)|D| .
$$

Proof of Theorem 2. It follows immediately from Lemmas 7 and 10.

We now set out to prove a converse result to Theorem 2.

Lemma 11. For every graph $G$, there is a graph $G^{\prime}$ containing $G$ as a minor, such that $\operatorname{mcr}(G)=\operatorname{cr}\left(G^{\prime}\right)$ and $\left|G^{\prime}\right| \leq|G|+\operatorname{mcr}(G)$.

Proof. By definition, there is a graph $G^{\prime}$ containing $G$ as a minor, such that $\operatorname{mcr}(G)=$ $\operatorname{cr}\left(G^{\prime}\right)$. Choose such a graph $G^{\prime}$ with the minimum number of vertices. There is a set $\left\{T_{v}: v \in V(G)\right\}$ of disjoint subtrees in $G^{\prime}$, such that for every edge $v w$ of $G$, some vertex of $T_{v}$ is adjacent to some vertex of $T_{w}$. Every vertex of $G^{\prime}$ is in some $T_{v}$, as otherwise we could delete the vertex from $G^{\prime}$. Hence

$$
\left|G^{\prime}\right|=\sum_{v \in V(G)}\left|T_{v}\right|=|G|+\sum_{v \in V(G)}\left(\left|T_{v}\right|-1\right)=|G|+\sum_{v \in V(G)}\left\|T_{v}\right\| .
$$

We can assume that every edge of every subtree $T_{v}$ is in some crossing, as otherwise we could contract the edge. Thus $\left|G^{\prime}\right| \leq|G|+\operatorname{cr}\left(G^{\prime}\right)=|G|+\operatorname{mcr}(G)$. 
The next lemma is an analogue of Lemma 8.

Lemma 12. Every graph $G$ has a planar decomposition with width 2 and order $|G|+$ $2 \operatorname{mcr}(G)$.

Proof. By Lemma 11, there is some graph $G^{\prime}$ containing $G$ as a minor, such that $\operatorname{cr}\left(G^{\prime}\right)=$ $\operatorname{mcr}(G)$ and $\left|G^{\prime}\right| \leq|G|+\operatorname{mcr}(G)$. By Lemma 8, $G^{\prime}$ has a planar decomposition of width 2 and order $\left|G^{\prime}\right|+\operatorname{cr}\left(G^{\prime}\right)=\left|G^{\prime}\right|+\operatorname{mcr}(G) \leq|G|+2 \operatorname{mcr}(G)$. By Lemma 3, $G$ has a planar decomposition with the same properties.

We have the following characterisation of graphs with linear minor crossing number, which is analogous to Theorem 3 for crossing number (without the dependence on degree).

Theorem 4. The following are equivalent for a graph $G$ :

1. $\operatorname{mcr}(G) \leq c_{1}|G|$ for some constant $c_{1}$,

2. $G$ has a planar decomposition with width $c_{2}$ and order $|G|$ for some constant $c_{2}$,

3. $G$ has a planar decomposition with width 2 and order $c_{3}|G|$ for some constant $c_{3}$.

Proof. Lemma 12 implies $(1) \Rightarrow(3)$. Lemma 4 implies that $(3) \Rightarrow(2)$. Lemma 10 implies that $(2) \Rightarrow(1)$.

\section{References}

[1] Jay Adamsson And R. Bruce Richter. Arrangements, circular arrangements and the crossing number of $C_{7} \times C_{n}$. J. Combin. Theory Ser. B, 90(1):21-39, 2004. MR2041316, Zbl 1033.05026.

[2] Miklós Ajtai, Vašek Chvátal, Monroe M. Newborn, and Endre SzeMERÉDI. Crossing-free subgraphs. In Theory and practice of combinatorics, vol. 60 of North-Holland Math. Stud., pp. 9-12. North-Holland, 1982. MR806962, Zbl 0502.05021 .

[3] Sandeep N. Bhatt and F. Thomson Leighton. A framework for solving VlSi graph layout problems. J. Comput. System Sci., 28(2):300-343, 1984. MR0760549.

[4] Drago Bokal. Infinite families of crossing-critical graphs with prescribed average degree and crossing number. 2006. Submitted.

[5] Drago Bokal. On the crossing number of cartesian products with trees. J. Graph Theory, 56(4):287-300, 2007.

[6] Drago Bokal. On the crossing numbers of cartesian products with paths. $J$. Combin. Theory Ser. B, 97(3):381-384, 2007.

[7] Drago Bokal, Éva Czabarka, lászló A. SzÉkely, and Imrich VrT̃o. Graph minors and the crossing number of graphs. Electron. Notes Discrete Math., 28:169-175, 2007. 
[8] Drago Bokal, GaŠper Fijavž, And Bojan Mohar. The minor crossing number. SIAM J. Discrete Math., 20(2):344-356, 2006. MR2257266.

[9] Károly Böröczky, János Pach, and GÉzA Tóth. Planar crossing numbers of graphs embeddable in another surface. Internat. J. Found. Comput. Sci., 17(5):10051015, 2006. MR2270948, Zbl 1100.68075.

[10] Reinhard Diestel and Daniela Kühn. Graph minor hierarchies. Discrete Appl. Math., 145(2):167-182, 2005. MR2113139, Zbl 1055.05140.

[11] Hristo N. Djidjev and Imrich VRT'O. Crossing numbers and cutwidths. J. Graph Algorithms Appl., 7(3):245-251, 2003. MR2112230, Zbl 1066.05054.

[12] Hristo N. Djidjev And ImRICh VRŤo. Planar crossing numbers of genus $g$ graphs. In Michele Bugliesi, Bart Preneel, Vladimiro Sassone, and Ingo Wegener, eds., Proc. 33rd International Colloquium on Automata, Languages and Programming (ICALP '06), vol. 4051 of Lecture Notes in Comput. Sci., pp. 419-430. Springer, 2006.

[13] Paul Erdős and Richard K. Guy. Crossing number problems. Amer. Math. Monthly, 80:52-58, 1973. MR0382006, Zbl 0264.05109.

[14] Enrique Garcia-Moreno and Gelasio Salazar. Bounding the crossing number of a graph in terms of the crossing number of a minor with small maximum degree. J. Graph Theory, 36(3):168-173, 2001. MR1814533, Zbl 0979.05033.

[15] Michael R. Garey and David S. Johnson. Crossing number is NP-complete. SIAM J. Algebraic Discrete Methods, 4(3):312-316, 1983. MR0711340, Zbl 0536.05016 .

[16] James F. Geelen, R. Bruce Richter, and Gelasio Salazar. Embedding grids in surfaces. European J. Combin., 25(6):785-792, 2004. MR2079898, Zbl 1050.05035 .

[17] Lev Yu. Glebsky and Gelasio Salazar. The crossing number of $C_{m} \times C_{n}$ is as conjectured for $n \geq m(m+1)$. J. Graph Theory, 47(1):53-72, 2004. MR2079290, Zbl 1053.05032.

[18] Petr Hliněny. Crossing-number critical graphs have bounded path-width. $J$. Combin. Theory Ser. B, 88(2):347-367, 2003. MR1983364, Zbl 1021.05028.

[19] Petr HliněnÝ. Crossing number is hard for cubic graphs. J. Combin. Theory Ser. B, 96(4):455-471, 2006. MR2232384, Zbl 1092.05016.

[20] F. Thomson Leighton. Complexity Issues in VLSI. MIT Press, 1983. ISBN 0262121042 .

[21] F. Thomson Leighton. New lower bound techniques for VLSI. Math. Systems Theory, 17(1):47-70, 1984. MR0738751, Zbl 0488.94048.

[22] Bernard Montaron. An improvement of the crossing number bound. J. Graph Theory, 50(1):43-54, 2005. MR2157537, Zbl 1073.05020.

[23] Nagi H. Nahas. On the crossing number of $K_{m, n}$. Electron. J. Combin., 10:N8, 2003. MR2014536, Zbl 1023.05039. 
[24] Seiya Negami. Crossing numbers of graph embedding pairs on closed surfaces. J. Graph Theory, 36(1):8-23, 2001. MR1803630, Zbl 0971.05037.

[25] János Pach, Radoš Radoičić, Gábor Tardos, and GÉza Tóth. Improving the crossing lemma by finding more crossings in sparse graphs. Discrete Comput. Geom., 36(4):527-552, 2006. MR2267545, Zbl 1104.05022.

[26] János Pach, Farhad Shahrokhi, and Mario Szegedy. Applications of the crossing number. Algorithmica, 16(1):111-117, 1996. MR1394496, Zbl 0851.68088.

[27] János Pach And Micha Sharir. On the number of incidences between points and curves. Combin. Probab. Comput., 7(1):121-127, 1998. MR1611057, Zbl 0901.52016.

[28] JÁnos Pach And GÉza Tóth. Thirteen problems on crossing numbers. Geombinatorics, 9(4):194-207, 2000. MR1763979.

[29] JÁnos PACH And GÉzA Tóth. Which crossing number is it anyway? J. Combin. Theory Ser. B, 80(2):225-246, 2000. MR1794693, Zbl 1023.05042.

[30] János PaCh And GÉza Tóth. Crossing number of toroidal graphs. In Patrick Healy and Nikola S. Nikolov, eds., Proc. 13th International Symp. on Graph Drawing (GD '05), vol. 3843 of Lecture Notes in Comput. Sci., pp. 334-342. Springer, 2006. MR2244523.

[31] Michael J. Pelsmajer, Marcus Schaefer, And Daniel Štefankovič. Crossing number of graphs with rotation systems. In Proc. 15th International Symp. on Graph Drawing (GD '07), Lecture Notes in Comput. Sci. Springer, to appear.

[32] Benny Pinontoan and R. Bruce Richter. Crossing numbers of sequences of graphs. II. Planar tiles. J. Graph Theory, 42(4):332-341, 2003.

[33] Benny Pinontoan and R. Bruce Richter. Crossing numbers of sequences of graphs. I. General tiles. Australas. J. Combin., 30:197-206, 2004.

[34] Helen Purchase. Which aesthetic has the greatest effect on human understanding? In Giuseppe Di Battista, ed., Proc. 5th International Symp. on Graph Drawing (GD '97), vol. 1353 of Lecture Notes in Comput. Sci., pp. 248-261. Springer, 1997.

[35] Helen C. Purchase. Performance of layout algorithms: Comprehension, not computation. J. Visual Languages and Computing, 9:647-657, 1998.

[36] Helen C. Purchase, Robert F. Cohen, and Murray I. James. An experimental study of the basis for graph drawing algorithms. ACM Journal of Experimental Algorithmics, 2(4), 1997. http://www.jea.acm.org/1997/PurchaseDrawing/.

[37] R. Bruce Richter and Gelasio Salazar. A survey of good crossing number theorems and questions. preprint.

[38] R. Bruce Richter And Jozef Širáñ. The crossing number of $K_{3, n}$ in a surface. J. Graph Theory, 21(1):51-54, 1996. MR1363689, Zbl 0838.05033.

[39] R. Bruce Richter and Carsten Thomassen. Intersections of curve systems and the crossing number of $C_{5} \times C_{5}$. Discrete Comput. Geom., 13(2):149-159, 1995. MR1314959, Zbl 0820.05015. 
[40] R. Bruce Richter and Carsten Thomassen. Relations between crossing numbers of complete and complete bipartite graphs. Amer. Math. Monthly, 104(2):131137, 1997. MR1437414, Zbl 0872.05010.

[41] Neil Robertson and Paul D. Seymour. Excluding a graph with one crossing. In Graph Structure Theory. Proceedings of the AMS-IMS-SIAM Joint Summer Research Conference on Graph Minors, vol. 147 of Contemp. Math., pp. 669-675. Amer. Math. Soc., 1993.

[42] Neil Robertson and Paul D. Seymour. Graph minors. II. Algorithmic aspects of tree-width. J. Algorithms, 7(3):309-322, 1986.

[43] Neil Robertson and Paul D. Seymour. Graph minors. XX. Wagner's conjecture. J. Combin. Theory Ser. B, 92(2):325-357, 2004. MR2099147, Zbl 1061.05088.

[44] Farhad Shahrokhi, Ondrej SÝkora, László A. Székely, and Imrich VRŤO. The crossing number of a graph on a compact 2-manifold. Adv. Math., 123(2):105-119, 1996. MR1420482, Zbl 0865.05036.

[45] Farhad Shahrokhi and László A. Székely. On canonical concurrent flows, crossing number and graph expansion. Combin. Probab. Comput., 3(4):523-543, 1994. MR1314073, Zbl 0817.05022.

[46] Farhad Shahrokhi, László A. Székely, Ondrej SÝkora, and Imrich VRŤO. Drawings of graphs on surfaces with few crossings. Algorithmica, 16(1):118 131, 1996. MR1394497, Zbl 0854.68074.

[47] LÁszló A. SzÉKely. Crossing numbers and hard Erdős problems in discrete geometry. Combin. Probab. Comput., 6(3):353-358, 1997. MR1464571, Zbl 0882.52007.

[48] LÁszló A. SzÉKelY. A successful concept for measuring non-planarity of graphs: the crossing number. Discrete Math., 276(1-3):331-352, 2004. MR2046646, Zbl 1035.05034 .

[49] LÁszló A. SzÉKely. Progress on crossing number problems. In Theory and Practice of Computer Science (SOFSEM 2005), vol. 3381 of Lecture Notes in Comput. Sci., pp. 53-61. Springer, 2005.

[50] ImRICH VRŤO. Crossing numbers of graphs: A bibliography. 2007. ftp://ftp.ifi.savba.sk/pub/imrich/crobib.pdf.

[51] David R. Wood and Jan Arne Telle. Planar decompositions and the crossing number of graphs with an excluded minor. New York J. Math., 13:117-146, 2007. 